\title{
Device Characteristics and Hot Carrier Lifetime Characteristics Shift Analysis by Carbon Implant used for $V_{\text {th }}$ Adjustment
}

\author{
Seong-Yeol Mun ${ }^{1}$, Seong-Jun Kang ${ }^{2}$, and Yang-Hee Joung ${ }^{2 *}$, Member, KIICE \\ ${ }^{1}$ Department of Foundries FAB, Globalfoundries Silicon Partners Pte. Ltd., Woodland 738406, Singapore \\ ${ }^{2}$ Department of Electrical and Semiconductor Engineering, Chonnam National University, Yeosu 550-749, Korea
}

\begin{abstract}
In this paper, a carbon implant is investigated in detail from the perspectives of performance advantages and side effects for the thick n-type metal-oxide-semiconductor field-effect transistor (n-MOSFET). Threshold voltage (Vth) adjustment using a carbon implant significantly improves the Vth mismatch performance in a thick (3.3-V) n-MOS transistor. It has been reported that a bad mismatch occurs particularly in the case of $0.11-\mu \mathrm{m}$ Vth node technology. This paper investigates a carbon implant process as a promising candidate for the optimal Vth roll-off curve. The carbon implant makes the Vth roll-off curve perfectly flat, which is explained in detail. Further, the mechanism of hot carrier injection lifetime degradation by the carbon implant is investigated, and new process integration involving the addition of a nitrogen implant in the lightly doped drain process is offered as its solution. This paper presents the critical side effects, such as Isub increases and device performance shifts caused by the carbon implant and suggests an efficient method to avoid these issues.
\end{abstract}

Index Terms: Carbon implant, Hot carrier injection, MOS transistor, Threshold voltage

\section{INTRODUCTION}

The decrease in the gate dimension has led to many new challenging barriers that need to be solved without a considerable trade off with the performance of other devices. Transistor $V_{\text {th }}$ mismatch is one of the critical parameters to investigate $[1,2]$. An improvement of the mismatch with decreasing gate oxide thickness is well documented, as is the relationship between the mismatch and the layout area, that is, mismatch $\propto 1 /(\mathrm{WL})^{1 / 2}[3]$.

The transistor $\mathrm{V}_{\text {th }}$ mismatch is more extreme in a transistor having a relatively thick gate oxide as implied by the abovementioned formula. Further, an n-type metal-oxidesemiconductor (n-MOS) transistor is known to be considerably weaker than a p-MOS transistor. The boron used for the $V_{\text {th }}$ adjustment in an n-MOS transistor has a high diffusion rate because of the thermal budget. That is the reason why the n-MOS transistor with thick gate oxide has the worst $V_{\text {th }}$ mismatch performance. Carbon prevents the boron from being diffused to the Si surface by trapping the interstitial Si through which the boron is diffused because of the thermal budget [4-6]. Carbon reacts with the interstitial Si according to the Watkins exchange mechanism [7], and then, the interstitial Si precipitates in the carbon-rich region. This carbon implant effect significantly reduces the thick n-MOS $\mathrm{V}_{\text {th }}$ mismatch in the $0.11-\mu \mathrm{m}$ technology when it is used in the thick n-MOS Vth adjustment implant step. However, carbon induces an extremely abrupt electric field in a lightly doped drain (LDD) junction resulting in hot carrier injection (HCI) lifetime degradation [8]. This paper explains the HCI degradation mechanism in detail and suggests the use of a nitrogen implant in the LDD step to improve the HCI lifetime.

Received 03 April 2013, Revised 22 July 2013, Accepted 09 August 2013

*Corresponding Author Yang-Hee Joung (E-mail: jyanghee@jnu.ac.kr, Tel: +82-61-659-7314)

Department of Electrical and Semiconductor Engineering, Chonnam National University, 50 Daehak-ro, Yeosu 550-749, Korea.

(C) This is an Open Access article distributed under the terms of the Creative Commons Attribution Non-Commercial License (http://creativecommons.org/li-censes/by$\mathrm{nc} / 3.0 /$ ) which permits unrestricted non-commercial use, distribution, and reproduction in any medium, provided the original work is properly cited. 
Further, a carbon implant is well known to deactivate the boron implanted in an n-type metal-oxide-semiconductor field-effect transistor (n-MOSFET) channel for Vth adjustment, resulting in a device performance shift [9]. This requires $\mathrm{Vth}_{\text {th }}$ implant tuning for a device performance match. The final process condition matching the device performance exhibits a perfectly flat $\mathrm{V}$ th roll-off curve without the HCI issue in a thick n-MOS. Thus far, no research has reported this phenomenon. Hence, its detail mechanism is explained in this paper.

Carbon implants have been extensively investigated for $\mathrm{V}$ th mismatch improvement. However, the side effects of the use of a carbon implant have not been investigated in detail [10]. This paper focuses on the unavoidable side effects on the transistor performance shift by the carbon implant, including their detail mechanisms. This paper suggests that the carbon implant should be combined with a critical process to prevent side effects. Finally, a number of characteristics of the carbon implant process are identified, and a clear process integration guideline for mass production is presented in this paper.

\section{EXPERIMENT}

A (100)-oriented p-type wafer with a resistivity of 9-12 $\Omega$-cm is cleaned using 1:19 diluted hydrogen fluoride, and a shallow trench isolation is formed. After well and threshold voltage ( $\mathrm{V}_{\text {th }}$ ) adjustment implantation, a carbon implant is added only in the thick n-MOSFET region $\left(2.0 \times 10^{14}, 10 \mathrm{keV}\right)$. The gate oxide is grown in a conventional furnace at $800^{\circ} \mathrm{C}$ by using a wet oxidation process targeting $68 \AA$ and $20 \AA$ for the high- and low-voltage areas, respectively. Following the gate oxide growth, undoped polysilicon is deposited at $530^{\circ} \mathrm{C}$ with $1,800 \AA$. Photolithography for gate patterning is performed at a line width of $0.11 \mu \mathrm{m}$ by using a $193-\mathrm{nm}$ deep ultraviolet (DUV) lithography technology after the application of organic bottom anti-reflective coating (BARC; DUV32J, Brewer Science, Rolla, MO, USA) and photoresist (PR; SR540, Stanford Research System, Sunnyvale, CA, USA) coating targeting $750 \AA$ and $7600 \AA$, respectively. A decoupled plasma source plasma etching reactor is used for BARC and the polysilicon gate etching process. Fig. 1 shows the detailed process schematic.

After gate poly formation, the LDD and the pocket implant are applied to the transistor. For a thick n-MOSFET, a nitrogen implant is added after the LDD implant with $\mathrm{N}_{2}$ $\left(5.0 \times 10^{14}, 25 \mathrm{keV}\right)$ to recover the HCI lifetime degradation caused by the carbon implant used in the $\mathrm{V}_{\text {th }}$ adjustment step.

A thick n-MOSFET is fabricated using the new process schematic and its electrical characteristics of the $\mathrm{V}_{\text {th }}$ mismatch, I-V curve, Isub curve, and $\mathrm{V}_{\text {th }}$ roll-off curve are determined using an Agilent HP4156 parameter analyzer.

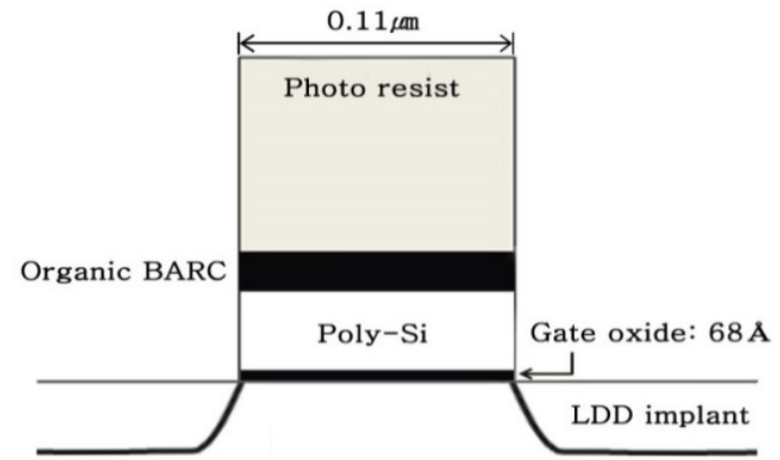

Fig. 1. Process schematic of the gate structure of a thick n-type metaloxide-semiconductor field-effect transistor with a carbon implant in the $V_{\text {th }}$ adjustment step and a nitrogen implant in the lightly doped drain (LDD) implant step. BARC: bottom anti-reflective coating.

\section{RESULTS AND DISCUSSION}

Theoretically, the MOSFET threshold voltage ( $\mathrm{V}_{\text {th }}$ variation and mismatch should be inversely proportional to the square root of the active transistor area as in (1) [3],

$$
\sigma V_{t h}=\frac{A_{V T}}{\sqrt{W L}}
$$

where $A_{V T}$ represents a technology conversion constant and $W L$ denotes the product of the transistor's active area.

The carbon implant used in the $\mathrm{V}_{\text {th }}$ adjustment implant step significantly improves the $\mathrm{V}$ th mismatch in a thick n-MOS of the $0.11-\mu \mathrm{m}$ technology from $18 \mathrm{mV}$ to $12 \mathrm{mV}$ as shown in Fig. 2.

However, carbon induces an extremely abrupt electric field in the LDD junction by reducing the boron diffusion in the LDD junction [11], which cannot be simulated due to the tool limitation. This increases the substrate current (Isub) as shown in Fig. 3, resulting in HCI lifetime degradation. This degradation is due to the same mechanism as that of

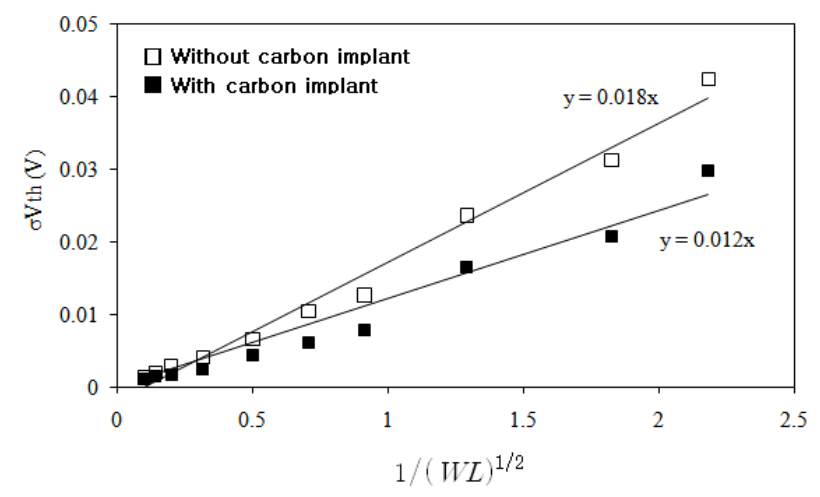

Fig. 2. Thick n-type metal-oxide-semiconductor field-effect transistor threshold voltage $\left(V_{t h)}\right.$ mismatch performance processed by the carbon implant in the $V$ th adjustment step with $\mathrm{C}\left(2.0 \times 10^{14}, 10 \mathrm{keV}\right)$. 


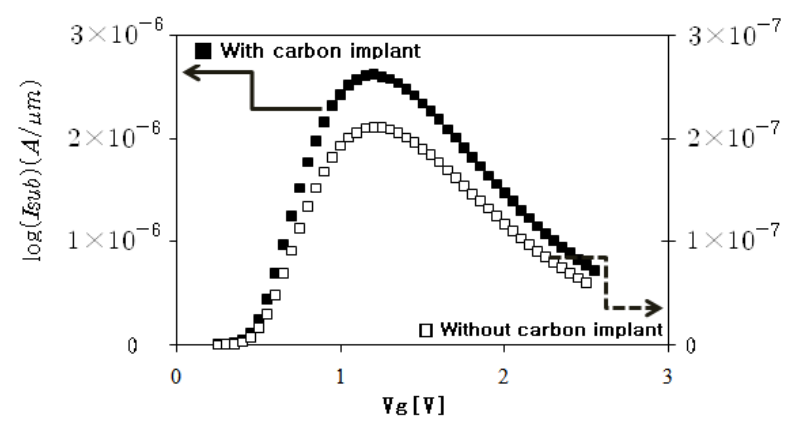

Fig. 3. Isub comparison curve between n-MOSFET with and without a carbon implant.

the carbon-implant-induced $V_{\text {th }}$ mismatch improvement by the prevention of boron diffusion in the channel region. The boron diffusion is suppressed in the LDD junction, causing an abrupt electric field profile in the LDD junction. The abrupt junction is known to increase the Isub, thereby degrading the HCI lifetime.

A nitrogen implant was applied in the LDD implant step to recover the graded junction from the abrupt junction caused by the carbon implant only in the LDD junction region without affecting the channel region. The nitrogen implant is known to improve the HCI lifetime by creating a graded electric field profile at the LDD junction. The inert

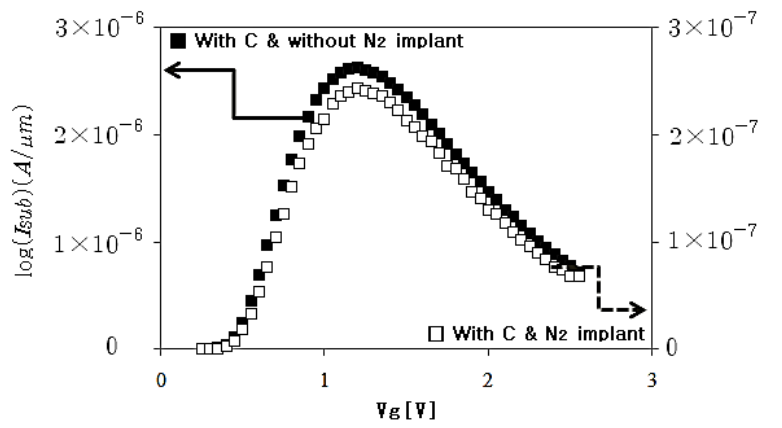

Fig. 4. Isub comparison curve between transistors with and without carbon implant and nitrogen implant $\left(5.0 \times 10^{14}, 25 \mathrm{keV}\right)$.

nitrogen leads to the formation of interstitial $\mathrm{Si}$, helping the boron in the LDD junction to diffuse well and resulting in a graded junction. The nitrogen implant $\left(5.0 \times 10^{14}, 25 \mathrm{keV}\right)$ is proven to reduce the Isub to the level of the without carbon implant process as shown in Fig. 4.

The carbon implant is known to deactivate the boron doped in the channel for the $\mathrm{V}$ th adjustment, resulting in a $\mathrm{V}$ th shift. Additional device tuning is carried out to recover this shift, as shown in Fig. 5.

The $V_{\text {th }}$ curve verified using the new process schematic shows a perfectly flat curve that was never expected, as shown in Fig. 6.
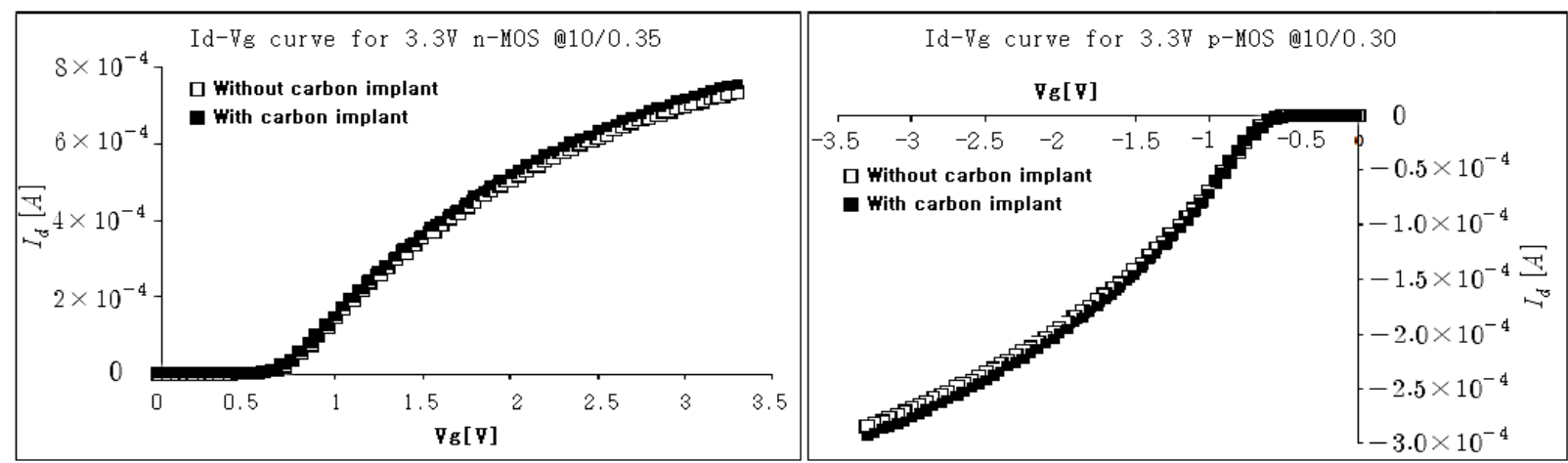

(a)
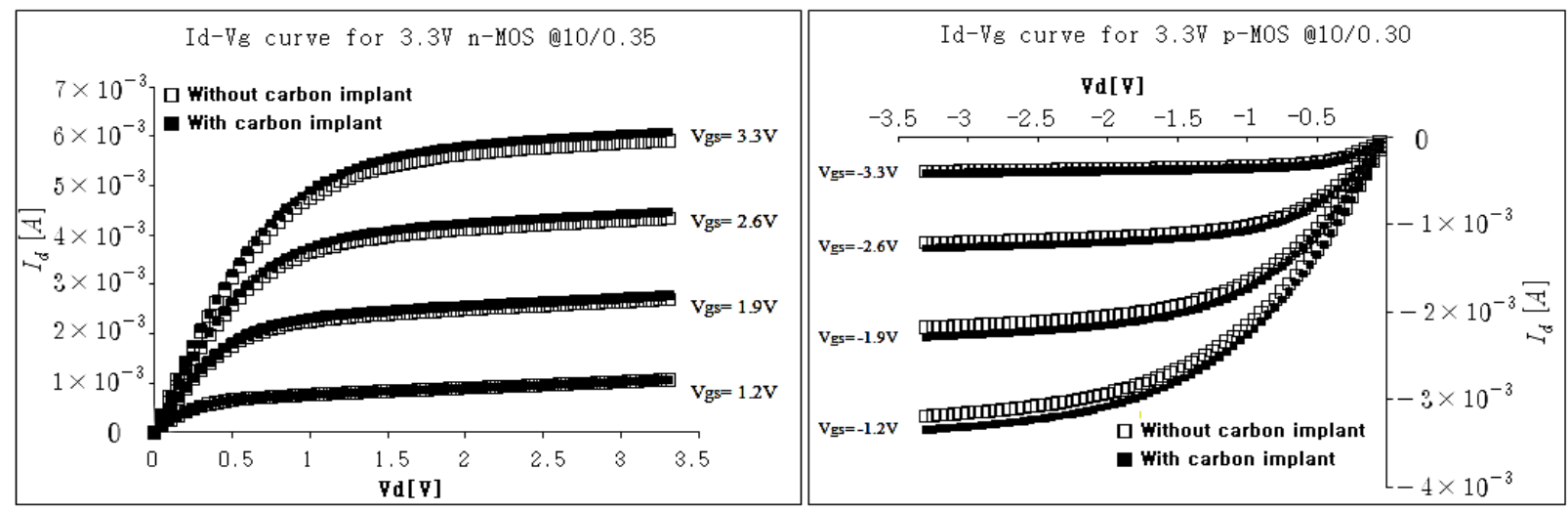

(b)

Fig. 5. $\mid-V$ curve comparison between transistors with and without a carbon and nitrogen implant: (a) $I_{d}-V_{g}$ curve, (b) $I_{d}-V_{g}$ curve. 


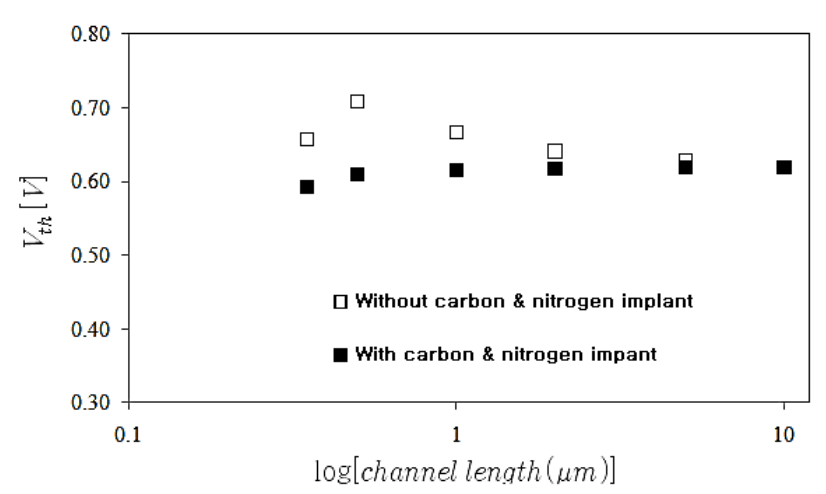

Fig. 6. Threshold voltage $\left(V_{t h}\right)$ roll-off curve comparison between n-MOSFET with and without carbon and nitrogen implants.

Therefore, we need to understand the mechanism of the non-flat $\mathrm{V}$ th roll-off curve for the case without the carbon implant in order to understand the estimated mechanism of the flat $V_{\text {th }}$ curve in the case of with the carbon implant. The divot area, the boundary between the isolation oxide and the active area, is known to have a lower $\mathrm{V}_{\text {th }}$ than the channel $\mathrm{V}$ th because the boron concentration in the divot area is lower than that in the channel. The lower $\mathrm{V}_{\text {th }}$ ' portion is large in the long channel; however, this portion decreases with a decrease in the channel length, resulting in a $V_{\text {th }}$ increase. However, the $\mathrm{V}$ th decreases again in a very short channel because of the short channel effect, resulting in a curve.

The carbon implant deactivates the boron as explained earlier, which is estimated to significantly reduce the $\mathrm{V}_{\text {th }}$ di-fference between the channel and the divot area. As a result, the contribution of the lower $\mathrm{V}$ th in the divot area is the same as that along a different channel length, resulting in a perfectly flat $V_{\text {th }}$ curve with respect to the channel length.

\section{CONCLUSIONS}

A carbon implant is investigated in detail from the perspectives of performance, advantages, and side effects for a thick (3.3-V) n-MOSFET. A carbon implant is very promising for transistor mismatching improvement with an ideal $\mathrm{V}_{\text {th }}$ roll-off curve. However, the carbon implant causes unavoidable side effects. This paper investigates critical issues, such as Isub increases and device performance shifts and suggests the use of a nitrogen implant and $\mathrm{V}$ th fine-tuning as solutions. The new process integration schematic involving the addition of a nitrogen implant in the LDD process recovers the Isub degradation and the device performance shift caused by the carbon implant well. Finally, this study also investigated the $\mathrm{V}_{\text {th }}$ roll-off curve shift by the new process integration schematic and recommends this new schematic as a very promising process integration method with a perfect $\mathrm{V}$ th roll-off curve device.

\section{REFERENCES}

[1] H. K. Jung, "Analysis of doping profile dependent threshold voltage for DGMOSFET using Gaussian function," Journal of Information and Communication Engineering, vol. 9, no. 3, pp. 310-314, 2011.

[2 ] H. K. Jung, "Analysis of subthreshold characteristics for device parameter of DGMOSFET using Gaussian function," Journal of Information and Communication Engineering, vol. 9, no. 6, pp. 733-737, 2011.

[ 3 ] M. J. M. Pelgrom, A. C. J. Duinmaijer, and A. P. G. Welbers, "Matching properties of MOS transistors," IEEE Journal of SolidState Circuits, vol. 24, no. 5, pp. 1433-1439, 1989.

[ 4 ] I. Ban, M. C. Ozturk, and E. K. Demirlioglu, "Suppression of oxidation-enhanced boron diffusion in silicon by carbon implantation and characterization of MOSFET's with carbonimplanted channels," IEEE Transactions on Electron Devices, vol. 44, no. 9, pp. 1544-1551, 1997.

[ 5 ] S. Rizk, Y. M. Haddara, and A. Sibaja-Hernandez, "Modeling the suppression of boron diffusion in $\mathrm{Si} / \mathrm{SiGe}$ due to carbon incorporation," Journal of Vacuum Science \& Technology B Microelectronics and Nanometer Structures, vol. 24, no. 3, pp. 1365-1370, 2006.

[6] M. S. Carroll, C. L. Chang, J. C. Sturm, and T. Buyuklimanli, "Complete suppression of boron transient-enhanced diffusion and oxidation-enhanced diffusion in silicon using localized substitutional carbon incorporation," Applied Physics Letters, vol. 73, no. 25, pp. 3695-3697, 1998.

[ 7 ] E. Rotem, J. M. Shainline, and J. M. Xu, "Electrolumine-scence of nanopatterned silicon with carbon implantation and solid phase epitaxial regrowth," Optics Express, vol. 15, no. 21, pp. 1409914106, 2007.

[ 8 ] W. Lee, S. Lee, T. Ahn, and H. Hwang, "Degradation of hot carrier lifetime for narrow with MOSFET with shallow trench isolation," in Proceedings of the IEEE International Reliability Physics Symposium, San Diego: CA, pp. 259-262, 1999.

[ 9 ] J. B. Johnson, T. B. Hook, and Y. M. Lee, "Analysis and modeling of threshold voltage mismatch for CMOS at $65 \mathrm{~nm}$ and beyond," IEEE Electron Device Letters, vol. 29, no. 7, pp. 802-804, 2008.

[10] H. S. Park, K. S. Kim, S. J. Park, S. J. Oh, J. S. Lee, and H. S. Park, "Impact of profiled LDD structure on hot carrier degradation of NMOSFET's," in Proceedings of the 26th European Solid State Device Research Conference, Bologna, Italy, pp. 991-994, 1996.

[11] C. F. Tan, J. G. Lee, L. W. Teo, C. Yin, G. Lin, E. Quek, and S. Chu, "A carbon co-implantation technique for formation of steep halo for nFET short channel effect improvement and performance boost," in Proceedings of the International Symposium on VLSI Technology, Systems and Applications, Hsinchu, Taiwan, pp. 32-33, 2008. 


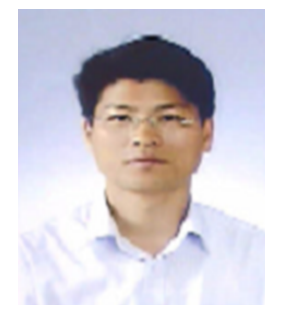

\section{Seong-Yeol Mun}

received his M.S. degree in 2006 and Ph.D. degree in 2011 in electrical and semiconductor engineering from Chonnam National University. He is presently working at Globalfoundries Silicon Partners Pte. Ltd., Singapore. His current research interests are process development and improvement of CMOS active pixel image sensors.

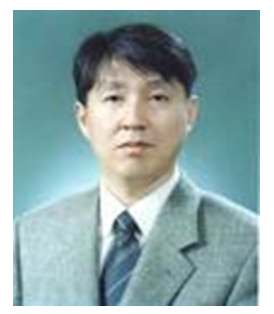

\section{Seong-Jun Kang}

received his M.S. degree in 1994 and Ph.D. degree in 1999 in electronic materials and devices engineering from Inha University. Since 2000, he has been working as Professor in the Department of Electrical and Semiconductor Engineering, Chonnam National University, Cheonnam, Korea. His current research interests include semiconductor processing and optical devices applications of oxide and II-VI compound semiconductors.

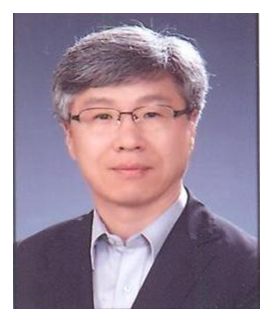

\section{Yang-Hee Joung}

received his M.S. degree in 1985 and Ph.D. degree in 1993 in electronic materials and devices engineering from Inha University. Since 1995, he has been working as Professor in the Department of Electrical and Semiconductor Engineering, Chonnam National University, Chonnam, Korea. His current research interests are semiconductor processing and semiconductor physics. 